PAPER

\title{
Clinical and imaging predictors of intracerebral haemorrhage in stroke patients treated with intravenous tissue plasminogen activator
}

\author{
L Derex, $M$ Hermier, P Adeleine, J-B Pialat, $M$ Wiart, Y Berthezène, F Philippeau, J Honnorat, \\ J-C Froment, P Trouillas, N Nighoghossian
}

J Neurol Neurosurg Psychiatry 2005;76:70-75. doi: 10.1136/jnnp.2004.038158

See end of article for authors' affiliations

.....................

Correspondence to: Dr Laurent Derex,

Service d'Urgences

Neurovasculaires, Hôpital

Neurologique, 59

boulevard Pinel, 69003

Lyon, France;

laurent.derex@

chu-lyon.fr

Received 30 January 2004

In revised form

6 April 2004

Accepted 6 April 2004

\begin{abstract}
Objective: To evaluate clinical, biological, and pretreatment imaging variables for predictors of tissue plasminogen activator (tPA) related intracerebral haemorrhage (ICH) in stroke patients.

Methods: 48 consecutive patients with hemispheric stroke were given intravenous $P$ PA within seven hours of symptom onset, after computed tomography (CT) and magnetic resonance imaging (MRI) of the brain. Baseline diffusion weighted (DWI) and perfusion weighted (PWI) imaging volumes, time to peak, mean transit time, regional cerebral blood flow index, and regional cerebral blood volume were evaluated. The distribution of apparent diffusion coefficient (ADC) values was determined within each DWI lesion.

Results: The symptomatic ICH rate was $8.3 \%$ (four of 48 ); the rate for any $\mathrm{ICH}$ was $43.8 \%$ (21 of 48 ). Univariate analysis showed that age, weight, history of hyperlipidaemia, baseline NIHSS score, glucose level, red blood cell count, and lacunar state on MRI were associated with ICH. However, mean 24 hour systolic blood pressure and a hyperdense artery sign on pretreatment CT were the only independent predictors of ICH. Patients with a hyperdense artery sign had larger pretreatment PWI and DWI lesion volumes and a higher NIHSS score. Analysis of the distribution of ADC values within DWI lesions showed that a greater percentage of pixels had lower ADCs $\left(<400 \times 10^{-6} \mathrm{~mm}^{2} / \mathrm{s}\right)$ in patients who experienced $\mathrm{ICH}$ than in those who did not.

Conclusion: Key clinical and biological variables, pretreatment CT signs, and MRI indices are associated with tPA related intracerebral haemorrhage.
\end{abstract}

$\mathrm{H}^{2}$ aemorrhagic transformation of a brain infarct is a major concern in acute stroke treatment. Spontaneous bleeding within the infarcted brain tissue is common, especially during the first week of ischaemic stroke. ${ }^{1-4}$ The frequency of haemorrhagic transformation is increased in stroke patients treated with anticoagulant therapy or thrombolysis..$^{5-7}$ Symptomatic intracerebral haemorrhage (ICH) is obviously the most feared complication of thrombolysis for stroke. Different clinical and radiological factors contributing to tissue plasminogen activator (tPA) related ICH have been reported previously, such as increasing age, severe neurological deficit on admission, and the importance of the extent of parenchymal hypoattenuation on baseline computed tomography (CT) $)^{5-7}$ The European cooperative acute stroke study (ECASS) investigators were first to suggest that the baseline CT data could be predictive of subsequent ICH. ${ }^{8}$ In the ECASS II trial, the incidence of parenchymal haematoma type 2, which independently causes clinical deterioration, was significantly more frequent with the presence of large early hypodensity on baseline CT. ${ }^{9}$ On the other hand, data on magnetic resonance imaging (MRI) markers of tPA related ICH remain limited. ${ }^{10-13}$ Pretreatment assessment of thrombolytic candidates with a clinical, biological, CT, and multimodal MRI screening could identify more predictors of $\mathrm{ICH}$. We present such a multiparametric evaluation of factors predicting tPA related $\mathrm{ICH}$.

\section{METHODS}

\section{Patients}

Consecutive patients with symptoms of acute internal carotid artery (ICA) territory stroke were recruited for this prospective study.

\section{Inclusion criteria were:}

- time from symptom onset to tPA administration less than seven hours;

- absence of haemorrhage on brain CT;

- no contraindications to MRI or thrombolysis (including previous ICH);

- informed consent obtained from the patient or authorised representative.

Neurological deficit was assessed on admission and at days 1,7 , and 60 by a neurologist certified in the use of the National Institutes of Health stroke scale (NIHSS). ${ }^{14} \mathrm{~A}$ significant neurological deficit (NIHSS score $\geqslant 4$ ) had to be present at the time of entry to the study. A modified Rankin scale (m-RS) score ${ }^{15}$ was obtained on day 60.

Intravenous alteplase (Actilyse ${ }^{\circledR}$, Boehringer Ingelheim, Biberach, Germany) was given according to a previously published protocol. ${ }^{16}$ Briefly, patients received tPA in a dose of $0.8 \mathrm{mg} / \mathrm{kg}$ body wt (maximum $90 \mathrm{mg}$ ), $10 \%$ of which was given as a bolus in the first minute, followed by delivery of the remaining $90 \%$ as a constant infusion over 90 minutes. An increase in blood pressure to more than $185 \mathrm{~mm} \mathrm{Hg}$ systolic or $110 \mathrm{~mm} \mathrm{Hg}$ diastolic after initiation of tPA was treated with intravenous nicardipine or labetalol. Systolic and diastolic blood pressure were recorded each hour during the

Abbreviations: ADC, apparent diffusion coefficient; ECASS, European cooperative acute stroke study; EIC, early ischaemic changes; EPI, echoplanar imaging; ICA, internal carotid artery; ICH, intracerebral haemorrhage; NIHSS, National Institutes of Health stroke scale; IPA, tissue plasminogen activator; TTP, time to peak 
first 24 hours. The study was reviewed and approved by the human research committee at our institution.

Demographic, clinical, laboratory, and imaging data for 46 variables (table l) were recorded for each patient. The diagnostic criteria for hypertension were systolic/diastolic blood pressure $\geqslant 140 / 90 \mathrm{~mm} \mathrm{Hg}$. The criteria for hyperlipidaemia were total cholesterol $\geqslant 5.18 \mathrm{mmol} / \mathrm{l}(200 \mathrm{mg} / \mathrm{dl})$, low density lipoprotein (LDL) $\geqslant 1.60 \mathrm{~g} / \mathrm{l}$, and/or triglycerides $\geqslant 2.26 \mathrm{mmol} / \mathrm{l}(200 \mathrm{mg} / \mathrm{dl})$.

The pretreatment $\mathrm{T}^{*}$ weighted MRI sequences were reviewed for evidence of old asymptomatic microbleeds (rounded, homogeneous, hypointense lesions $<5 \mathrm{~mm}$ in size $\left.^{17}\right)$. Symmetrical hyposignal of the globus pallidus, indicative of calcification, and loss of signal in the cerebral arteries, indicative of thrombus, were reasons for exclusion. Lacunes were defined as subcortical infarcts 3 to $20 \mathrm{~mm}$ in size on $\mathrm{T}^{*}$ weighted MRI sequences. ${ }^{18}$ The presence of leukoaraiosis on $\mathrm{T}^{*}$ weighted MRI sequences was assessed using the Fazekas rating scale. ${ }^{19}$

The TOAST ( trial of Org 10172 in acute stroke) criteria were used to classify stroke aetiology. ${ }^{20}$

\section{CT imaging and analyses}

All patients underwent non-contrast CT (with a fourth generation CT scanner) before treatment. Five millimetre thick contiguous slices were produced over the whole intracranial cavity. A second scan was done at (mean (SD)) 24 (6) hours, and a third scan on day 7; a control scan was obtained at any time when neurological deterioration was observed.

The study neuroradiologist $(\mathrm{MH})$, blinded to the clinical data and the results of previous or subsequent CT imaging,

Table 1 Demographic, clinical, laboratory, CT, and MRI variables

\begin{tabular}{ll}
\hline $\begin{array}{l}\text { Demographic and clinical } \\
\text { variables }\end{array}$ & CT/MRI indices \\
\hline Age & Lacunes \\
Sex & Leukoaraiosis \\
Weight & Early ischaemic changes \\
NIHSS score & Hyperdense artery sign \\
Systolic blood pressure & DWI lesion volume \\
Diastolic blood pressure & Mean ADC \\
Mean 24-hour SBP & PWI lesion volume \\
Mean 24-hour DBP & TTP \\
Hypertension on admission & RMTT \\
Glucose & rCBV \\
Cholesterol & rCBFi \\
C reactive protein & Microbleeds \\
White blood cell count & Presence of arterial occlusion \\
Red blood cell count & Site of occlusion \\
Platelet count & Recanalisation \\
Packed cell volume & Aetiology of stroke \\
International normalised ratio & \\
Cardioembolic source & \\
Partial thromboplastin time & \\
Fibrinogen & \\
Treatment delay & \\
Cigarette smoking & \\
Alcohol abuse & \\
Hypertension & \\
Diabetes & \\
Hyperlipidaemia & \\
Coronary artery disease & \\
Peripheral vascular disease & \\
Cerebrovascular diseases & \\
Current use of antiplatelets & \\
\hline & \\
& \\
& \\
& \\
& \\
& \\
&
\end{tabular}

$A D C$, apparent diffusion coefficient; $C T$, computed tomography; DBP, diastolic blood pressure; DWI, diffusion weighted imaging; MRI, magnetic resonance imaging; $\mathrm{PWI}$, perfusion weighted imaging; $\mathrm{RMT}$, regional mean transit time; $\mathrm{rCBFI}$, relative cerebral blood flow index; rrCBV, relative regional cerebral blood volume; SBP, systolic blood pressure; TTP, time to peak. evaluated each scan. ICH was defined according to the National Institute of Neurological Disorders and Stroke (NINDS) tPA stroke study and the ECASS-II criteria. ${ }^{5}$ Symptomatic ICH was defined as CT documented haemorrhage that was temporally related to neurological deterioration. Neurological deterioration was defined according to the PROACT II (prolyse in acute cerebral thromboembolism) criteria: at least a four point worsening on the NIHSS or a one point worsening on the NIHSS level of consciousness (item la), compared with the previous examination. ${ }^{21}$

All scans were examined for lacunes, the hyperdense artery sign, and early ischaemic changes (EIC). The volume of EIC was assessed prospectively (for example, $>33 \%$ of the MCA territory), as for ECASS. ${ }^{7}$ The hyperdense artery sign was defined as an area of increased attenuation, compared with the contralateral side of the brain, along the course of a cerebral artery, which was not consistent with atheromatous vessel wall calcification.

\section{MRI methods}

MRI was done at presentation and on day 1 after stroke onset using a $1.5 \mathrm{~T}$ whole body MR imager (Siemens AG, Erlangen, Germany), equipped with enhanced gradient hardware for echo-planar imaging (EPI). MRI protocol parameters have already been published. ${ }^{22}$ Sequences were always done in the same order: (1) time of flight turbo magnetic resonance angiography; (2) T2* weighted gradient echo (GE) sequence; (3) EPI isotropic diffusion weighted imaging (DWI); (4) perfusion weighted MRI (PWI) performed with GE EPI, using bolus passage of contrast agent.

\section{Data collection}

Various MRI indices-including baseline DWI and PWI lesion volumes-were determined by a senior neuroradiologist who was unaware of the clinical data. DWI and PWI indices were measured by placing regions of interest (ROI) within the DWI and PWI lesions manually.

To define DWI lesion volume, we used the trace images obtained at the highest $b$ value $\left(b=1000 \mathrm{~s} / \mathrm{mm}^{2}\right)$. The global lesion volume was determined by multiplying the area of diffusion hyperintensity by the sum of the slice thickness and the interslice gap thickness. Apparent diffusion coefficient (ADC) maps were generated by software using three values of $b\left(b=50, b=500\right.$, and $\left.b=1000 \mathrm{~s} / \mathrm{mm}^{2}\right)$. Pixel-by-pixel ADC values within each DWI lesion were grouped into 11 distinct classes $\left(<250 \times 10^{-6} \mathrm{~mm}^{2} / \mathrm{s}\right.$ to $\left.>1150 \times 10^{-6} \mathrm{~mm}^{2} / \mathrm{s}\right)$. The percentage of pixels within each class was determined. The mean lesional ADC value was calculated for each patient.

Perfusion maps were generated from the concentrationtime curve. A gamma variate fit was used on a pixel-by-pixel basis to compute parameter images for absolute time-to-peak (TTP) of signal drop. ${ }^{22}$ The PWI lesion volume was measured on the TTP maps (manual contouring). The global PWI lesion volume was determined by multiplying the area of perfusion abnormality by the sum of the slice thickness and the interslice gap thickness.

The evaluation of haemodynamic status also included relative regional cerebral blood volume ( $\mathrm{rCBV}$ ), assessed as the area under the concentration-time curve, relative mean transit time (rMTT), assessed as the first moment of the concentration-time curve, and regional cerebral blood flow index ( $\mathrm{rCBFi})$, assessed as the ratio rrCBV to rMTT within the perfusion deficit and the normal appearing contralateral mirror area. ${ }^{23}$

Vessel occlusions were categorised on magnetic resonance angiography (MRA) as proximal (ICA and Ml segment of the middle cerebral artery (MCA)) or distal (M2 segment of the MCA, distal branches of the MCA, and anterior cerebral 
artery (ACA)). Recanalisation was assessed on day l MRA (no recanalisation versus partial or full recanalisation).

\section{Reproducibility measurement}

For the DWI and PWI lesion volume measurements, two experienced observers measured the lesion on two occasions and the mean value was used. The interobserver and intraobserver variation in measurements was assessed by comparing the differences in measurements for the 48 cases. The intraobserver and interobserver reliability $(r)$ was $r=0.95$, with a mean deviation of less than $5 \%$ for intraobserver reproducibility. The intraobserver and interobserver reliabilities for lacune, leukoaraiosis, and microbleed detection on $\mathrm{T}^{*}$ sequences were $r=0.80,0.80$, and 0.90 , respectively. The intraobserver and interobserver reliability for the hyperdense artery sign detection on baseline CT scan was $r=0.85$.

\section{Statistical methods}

Quantitative data are expressed as mean (SD). We used Fisher's exact test to compare two binary variables and Pearson's $\chi^{2}$ test to compare two categorical variables in order to assess the significance of the association of pairs of noncontinuous variables. We applied the linear by linear association test for two ordinal variables. Spearman's rank order correlation coefficient was calculated for pairs of ordinal, continuous, and mixed (one ordinal and one continuous) variables. We applied the two sample $t$ test for one continuous variable and a binary factor (two groups). Multivariable analysis of the predictors of ICH was undertaken using a logistic regression model. The criterion for selecting explanatory variables was liberal $(p<0.20)$. Differences were considered statistically significant at $\mathrm{p}<0.05$. SPSS 10.0 (SPSS Inc, Chicago, Illinois, USA) was used for all calculations.

\section{RESULTS}

Between March 2001 and October 2002, MRI was undertaken in 94 consecutive ischaemic stroke patients admitted to our stroke unit. This represents $19 \%$ of patients admitted to hospital during the same period. Forty eight of these 94 patients ( 25 men and 23 women) underwent MRI before tPA. Their mean (SD) age was 63.8 (13.6) years. The mean NIHSS score was 13.7 (5.8). Time from stroke onset to CT was 2.68
(1.13) hours; time from stroke onset to MRI was 3.52 (1.27) hours. Time from stroke to tPA was 4.35 (1.20) hours.

Twenty patients $(41.7 \%)$ had a hyperdense artery sign on baseline CT: dense ICA sign in seven patients, dense MCA sign in 12 patients, and dense insular artery sign in three patients. Pretreatment MRA showed vessel occlusion in 46 patients, as follows: ICA+M1 MCA in 11; M1 MCA in 18; M2 and distal branches of the MCA in 15; ACA in two. Mean pretreatment DWI lesion volume was $47.5(59.3) \mathrm{cm}^{3}$; mean PWI lesion volume was 110.9 (56.8) $\mathrm{cm}^{3}$; mean ADC was $772.9(129.3) \times 10^{-6} \mathrm{~mm}^{2} / \mathrm{s}$.

The rate for symptomatic haemorrhage was $8.3 \%$ (four of 48). None of these four patients died. The rate for any haemorrhage was $43.8 \%$ (21 of 48 ). According to the NINDS criteria, six patients had intracerebral haematomas and 15 had haemorrhagic cerebral infarcts. According to the ECASS-II criteria, six patients had parenchymal haemorrhages $(\mathrm{PH} \mathrm{l}$ in two cases and PH 2 in four cases) and 15 had haemorrhagic infarcts (HI 1 in 12 cases and HI 2 in three cases).

No recurrent stroke was observed. Death occurred in four patients $(8.3 \%)$ during a two month follow up. Stroke aetiology was classified as follows, according to the TOAST criteria: 22 patients with large vessel disease, 18 with a cardioembolic source, four with small vessel disease, and four with cryptogenic stroke.

Single explanatory variable analysis of patient characteristics, biological variables, and imaging parameters predicting ICH are presented in tables 2 and 3. Age, weight, history of hyperlipidaemia, baseline NIHSS score, admission glucose level, red blood cell count, mean 24 hour systolic blood pressure, and lacunar state on $\mathrm{T} 2{ }^{*}$ were all associated with ICH.

Multivariable analysis of the predictors of ICH is presented in table 4. Mean 24 hour systolic blood pressure and a hyperdense artery sign on pretreatment CT were independent predictors of ICH. We measured the area under the receiver operating characteristic (ROC) curve to assess the prognostic power of our logistic regression model (fig 1). The model's ability to discriminate patients who experienced ICH from those who did not was excellent (area under the ROC curve $=0.82$; number of observations $=48$ ).

The correlations between clinical, CT, and MRI parameters and the hyperdense artery sign are presented in table 5 . Patients with a hyperdense artery sign had larger baseline

Table 2 Single explanatory variable analysis of demographic, clinical, and biological predictors of intracerebral haemorrhage

\begin{tabular}{|c|c|c|c|c|}
\hline Variables & HT group $(n=21)$ & $\begin{array}{l}\text { Non-HT group } \\
(n=27)\end{array}$ & Total & p Value \\
\hline Age (years) & $68.9(8.8)$ & $59.8(15.4)$ & $63.8(13.6)$ & 0.01 \\
\hline Weight $(\mathrm{kg})$ & 75.5 (14.7) & 64.7 (13.9) & $69.6(15.1)$ & 0.01 \\
\hline History of hypertension (\%) & 66.7 & 44.4 & 54.2 & 0.15 \\
\hline History of hyperlipidaemia (\%) & 52.4 & 22.2 & 35.4 & 0.04 \\
\hline History of atheroma (\%) & 57.1 & 29.6 & 41.7 & 0.08 \\
\hline NIHSS score & $15.5(5.6)$ & $12.2(5.6)$ & $13.7(5.8)$ & 0.05 \\
\hline Mean 24 hour SBP (mm Hg) & $151.8(16.0)$ & $134.2(18.9)$ & $142.1(19.5)$ & 0.001 \\
\hline Mean 24 hour DBP (mm Hg) & $81.9(8.9)$ & $76.7(10.8)$ & $79.0(10.2)$ & 0.08 \\
\hline Glucose (mmol/l) & $7.8(2.2)$ & $6.4(2.6)$ & $7.0(2.5)$ & 0.05 \\
\hline Platelet count $\left(\times 10^{9} / \mathrm{I}\right)$ & $225.8(59.8)$ & $243.3(68.5)$ & $235.6(64.8)$ & 0.36 \\
\hline $\begin{array}{l}\text { White blood cell count } \\
\left(\times 10^{9} / \mathrm{I}\right)\end{array}$ & $8.5(2.4)$ & $8.7(2.5)$ & $8.6(2.4)$ & 0.77 \\
\hline $\begin{array}{l}\text { Red blood cell count } \\
\left(\times 10^{12}\right.\end{array}$ & $46(0.4)$ & $4310.5)$ & $4.5(0.5)$ & 0.04 \\
\hline Cholesterol (mmol/l) & $5.3(1.0)$ & $5.2(1.0)$ & $5.2(1.0)$ & 0.83 \\
\hline Fibrinogen (g/l) & $3.6(0.6)$ & $3.3(0.9)$ & $3.5(0.8)$ & 0.23 \\
\hline Delay to $\mathrm{APA}$ (min) & $206(20)$ & $272(18)$ & $261(72)$ & 0.24 \\
\hline
\end{tabular}

Values are mean (SD) unless indicated otherwise. Significant values in bold.

DBP, diastolic blood pressure; HT, haemorrhagic transformation; NIHSS, National Institutes of Health stroke scale; $\mathrm{SBP}$, systolic blood pressure; $\mathrm{PAA}$, tissue plasminogen activator. 
Table 3 Single explanatory variable analysis of CT and MRI predictors of intracerebral haemorrhage

\begin{tabular}{|c|c|c|c|c|}
\hline Variables & HT group $(n=21)$ & Non-HT group $(n=27)$ & Total & p Value \\
\hline $\mathrm{EIC}(\%)$ & 76.2 & 63 & 68.8 & 0.37 \\
\hline EIC>1/3 MCA territory (\%) & 19 & 3.7 & 10.4 & 0.15 \\
\hline Hyperdense artery sign (\%) & 57.1 & 29.6 & 41.7 & 0.08 \\
\hline DWI lesion volume $\left(\mathrm{cm}^{3}\right)$ & $58.3(65.0)$ & $39.0(54.2)$ & $47.5(59.3)$ & 0.28 \\
\hline $\operatorname{ADC}\left(\times 10^{-6} \mathrm{~mm}^{2} / \mathrm{s}\right)$ & $760.2(142.2)$ & $783.2(119.7)$ & $772.9(129.3)$ & 0.55 \\
\hline PWI volume $\left(\mathrm{cm}^{3}\right)$ & $123.6(48.4)$ & $102(61.4)$ & $110.9(56.8)$ & 0.19 \\
\hline $\operatorname{TTP}(\mathrm{s})$ & $25.6(7.1)$ & $24.6(4.5)$ & $25.0(5.7)$ & 0.55 \\
\hline rMTT (s) & $14.4(4.7)$ & $14.4(2.8)$ & $14.4(3.7)$ & 0.95 \\
\hline $\mathrm{rCBFI}$ & $4.5(2.5)$ & $5.6(2.4)$ & $5.1(2.5)$ & 0.14 \\
\hline $\mathrm{rrCBV}$ & $33.4(13.7)$ & $38.2(11.7)$ & $36.1(12.7)$ & 0.20 \\
\hline Lacunes on T2* (\%) & 57.1 & 22.2 & 37.5 & 0.02 \\
\hline $\mathrm{MB}$ on $\mathrm{T}^{*}(\%)$ & 33.3 & 11.1 & 20.9 & 0.08 \\
\hline Recanalisation day 1 MRA (\%) & 50 & 55.6 & 52.1 & 0.32 \\
\hline
\end{tabular}

Values are mean (SD) unless indicated otherwise.

$\mathrm{ADC}$, apparent diffusion coefficient; $\mathrm{CT}$, computed tomography; DWI, diffusion weighted imaging; EIC, early ischaemic changes; $\mathrm{HT}$, haemorrhagic transformation; MB, microbleeds; MCA, middle cerebral artery; MRA, magnetic resonance angiography; MRI, magnetic resonance imaging; PWI, perfusion weighted imaging; rCBFI, relative cerebral blood flow index; rMTT, regional mean transit time; rrCBV, relative regional cerebral blood volume; TTP, time to peak.

PWI and DWI lesion volumes, a more severe initial neurological deficit, and a worse outcome.

The frequency distribution of ADC values within pretreatment DWI lesions is presented in fig 2. A greater percentage of pixels had lower ADCs $\left(<400 \times 10^{-6} \mathrm{~mm}^{2} / \mathrm{s}\right)$ in patients who experienced ICH than in patients who did not.

\section{DISCUSSION}

Multimodal MRI has the potential to select patients with evidence of salvageable brain tissue beyond the three hour time window. ${ }^{24}$ However, although meta-analyses of tPA given within the three to six hour time window shows that it has some efficacy, the benefit is smaller and the risk of bleeding is greater than in the optimal three hour time window. ${ }^{25}$ Thus the identification of predictors of ICH may improve patient selection and could lead to the safer use of thrombolysis beyond three hours.

Recent studies suggest that diffusion weighted MRI can help to identify patients at increased risk of $\mathrm{ICH}^{10-12}$ Our study represents the largest series so far in which ADC was calculated for each pixel in the whole ischaemic area to determine the influence of ADC values on the risk of ICH. We observed a significant influence of pretreatment ADC values on the bleeding risk. A greater percentage of pixels had lower ADCs $\left(<400 \times 10^{-6} \mathrm{~mm}^{2} / \mathrm{s}\right)$ in patients who experienced ICH than in patients who did not. Tong et al had already shown that ischaemic lesions destined to result in haemorrhagic transformation (HT) had lower ADC values than non-HT destined regions. ${ }^{10}$ Selim et al have also shown that the absolute volume of ischaemic tissue with ADC values below a cut off of $550 \times 10^{-6} \mathrm{~mm}^{2} / \mathrm{s}$ was associated with haemorrhagic transformation after tPA. ${ }^{12}$ Our results are consistent with these findings, with a lower cut off value. Careful analysis of the pretreatment ADC values could be helpful for identifying patients at increased risk of ICH. However, the feasibility of pretreatment ADC analysis should be examined prospectively in future studies. Additional MRI evaluation of blood-brain barrier disruption is also needed for a better assessment of the ischaemic damage to brain microvessels that contributes to the risk of haemorrhagic transformation after late thrombolysis with tPA. ${ }^{26}$ We were unable to detect any significant influence of the baseline DWI and PWI lesion volumes, TTP, rMTT, rCBFi, or rrCBV on the risk of ICH.

One limitation of our study is the use of relative haemodynamic indices. Accordingly, absolute quantification of $\mathrm{CBF}$ is needed before treatment to determine the haemodynamic threshold for risk of haemorrhagic transformation. Ueda et al have assessed cerebral blood flow in the territory of occluded vessels by single photon emission computed tomography (SPECT) in 20 stroke patients who underwent intra-arterial thrombolysis. ${ }^{27}$ Residual blood flow was significantly lower in the five patients who experienced ICH than in the 15 who did not.

The multivariable analyses in our study showed that the presence of a hyperdense artery sign on pretreatment CT was an independent predictor of ICH. The hyperdense middle cerebral artery sign is a well known predictor of occlusion of that artery with subsequent development of a large infarct and with a poor clinical outcome, even when patients are given tPA within three hours. ${ }^{28}{ }^{29}$ In our series, patients with a hyperdense artery sign had a more severe neurological deficit, a worse clinical outcome, and a specific pretreatment MRI pattern with larger DWI and PWI lesion volumes. The vast majority of patients with internal carotid artery or middle cerebral artery occlusion have poor collateral blood flow with a subsequent low flow state and increased
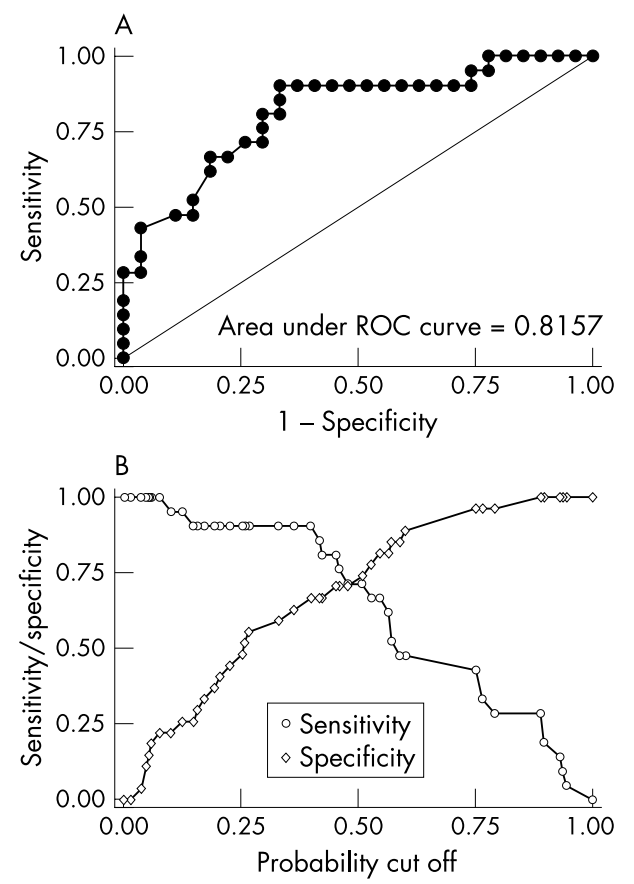

Figure 1 Receiver operating characteristic (ROC) curves assessing prognostic power of 24 hour systolic blood pressure plus a hyperdense artery sign in relation to intracerebral haemorrhage. 
Table 4 Multivariate analysis of prediction of intracranial haemorrhage $(n=48)$

\begin{tabular}{|c|c|c|c|c|c|}
\hline Variable & $\begin{array}{l}\text { Regression } \\
\text { coefficient }\end{array}$ & SE & $\begin{array}{l}\text { Estimated } \\
\text { odds ratio }\end{array}$ & $95 \% \mathrm{Cl}$ & p Value \\
\hline Mean 24 hour SBP & 0.075 & 0.025 & 1.078 & 1.027 to 1.131 & 0.003 \\
\hline $\operatorname{HAS}(0=$ no; $1=$ yes $)$ & 1.892 & 0.822 & 6.630 & 1.323 to 33.235 & 0.02 \\
\hline Constant & -11.772 & 3.783 & - & - & 0.002 \\
\hline \multicolumn{6}{|c|}{$\begin{array}{l}\text { Adjusted } R^{2} \text { (Nagelkerke) }=0.42 \text {; area under the } R O C \text { curve }=0.82 \text {. } \\
\text { The two explanatory variables (mean } 24 \text { hour systolic blood pressure and a hyperdense artery sign) plus the } \\
\text { constant represent the simplest adequate model. } \\
\mathrm{Cl} \text {, confidence interval; HAS, hyperdense artery sign; SBP, systolic blood pressure. }\end{array}$} \\
\hline
\end{tabular}

haemoglobin concentration, leading to an increased attenuation on CT. ${ }^{30}$ These patients with a proximal occlusion experience an immediate fall in blood flow in the territories supplied by the lenticulostriatal arteries, where collaterals are limited, leading to an increased risk of haemorrhagic transformation where ischaemic injury is greatest. ${ }^{31}$

With respect to clinical variables, our data corroborate previous studies showing that increasing age is associated with an increased risk of tPA related ICH. ${ }^{5-7}{ }^{32}$ In our study, the presence of lacunes on MRI was associated with an increased risk of ICH. Only a non-significant trend towards a higher risk of bleeding was detected for patients with microbleeds on $\mathrm{T}^{*}$ sequences. Other studies have shown that elderly patients with evidence of bleeding-prone microangiopathy are at higher risk of ICH under antithrombotic therapy. ${ }^{33}{ }^{34}$ High blood pressure during the first 24 hours was an independent predictor of ICH in our study. Raised blood pressure has already been related to ICH following thrombolysis for stroke..$^{532}$ These data highlight the importance of a careful monitoring of blood pressure within the first 24 hours.

Our results are consistent with previous findings suggesting an association between baseline serum glucose and tPA related $\mathrm{ICH}^{32}{ }^{35} 36$ There is experimental evidence that hyperglycaemia has a deleterious effect on ischaemic brain damage. In animal models, hyperglycaemia has been found to exaggerate blood-brain barrier injury, resulting in haemorrhagic transformation of the cerebral infarct. ${ }^{37} 38$ Thrombolytics are matrix metalloproteinases that may aggravate ischaemia induced damage to the basal lamina with subsequent loss of vessel wall integrity followed by haemorrhage. $^{38}$ This deleterious effect of tPA may be potentiated in the presence of hyperglycaemia.

Table 5 Correlations between pretreatment CT, MRI, clinical variables, and hyperdense artery sign on baseline CT (Spearman's $\rho$ )

\begin{tabular}{lcc}
\hline MRI indices & $\begin{array}{c}\text { Correlation } \\
\text { coefficient }\end{array}$ & $\mathbf{p ~ V a l u e ~}$ \\
\hline DWI lesion volume $\left(\mathrm{cm}^{3}\right)$ & 0.57 & $<\mathbf{0 . 0 0 0 1}$ \\
ADC $\left(10^{-6} \mathrm{~mm}^{2} / \mathrm{s}\right)$ & -0.14 & 0.33 \\
PWI lesion volume $\left(\mathrm{cm}^{3}\right)$ & 0.51 & $<0.0001$ \\
TTP $(\mathrm{s})$ & 0.003 & 0.98 \\
rrCBV & -0.04 & 0.77 \\
RCBFI & -0.20 & 0.18 \\
RMTT (s) & -0.01 & 0.95 \\
EIC $(\%)$ & 0.23 & 0.11 \\
NIHSS score & 0.56 & $<0.0001$ \\
Day 60 m-RS score & 0.42 & $\mathbf{0 . 0 0 4}$ \\
\hline
\end{tabular}

ADC, apparent diffusion coefficient; CT, computed tomography; DWI, diffusion weighted imaging; EIC, early ischaemic changes; MRI, magnetic resonance imaging; $m$-RS, modified Rankin scale; NIHSS, National Institutes of Health stroke scale; PWI, perfusion weighted imaging; $\mathrm{rCBFI}$, relative cerebral blood flow index; $\mathrm{rMT}$, regional mean transit time; rrCBV, relative regional cerebral blood volume; TTP, time to peak.

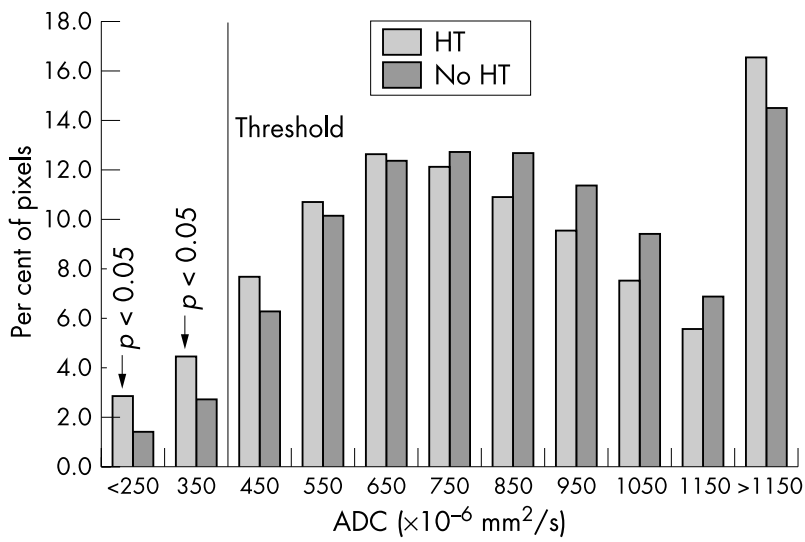

Figure 2 Frequency distribution of apparent diffusion coefficient (ADC) values within lesions identified on diffusion weighted imaging. HT, haemorrhagic transformation.

The present monocentre study has several potential limitations. Because there were only four symptomatic haemorrhages and four PH2 haematomas, we could not carry out a separate study of the factors predicting severe ICH. The spectrum of haemorrhagic transformation following tPA administration differs widely, encompassing trivial haemorrhagic petechiae and severe haematoma with a significant space occupying effect such as PH2 haematomas.' However, some investigators consider that the difference between symptomatic and asymptomatic haemorrhages may be related more to the degree of bleeding than to differences in pathophysiology. ${ }^{31} 39$ More information is needed on the predictors of tPA related severe haemorrhagic transformation and will probably come from multicentre studies, as the absolute number of severe tPA related cerebral haemorrhages is small. The lack of statistical power may also have prevented us from detecting any influence of early ischaemic CT changes on the cerebral bleeding risk following tPA administration.

\section{Conclusions}

This prospective, exhaustive, and homogeneous study shows that key clinical and biological variables, pretreatment CT signs, and MRI indices are predictors of tPA related ICH. The critical combination of these different factors may strongly influence the risk of bleeding. The next step would be to validate a multiparametric model predicting tPA related ICH in a larger cohort of patients with acute ischaemic stroke.

\section{ACKNOWLEDGEMENTS}

We thank professor Joseph Broderick, Stroke Research Center, University of Cincinnati, for critical review of the manuscript. We also thank Michèle Canova for her help in collecting and processing the data. 


\section{Authors' affiliations}

L Derex, F Philippeau, J Honnorat, Trouillas, N Nighoghossian, Service d'Urgences Neurovasculaires, Hôpital Neurologique, Lyon, France

P Adeleine, Service de Biostatistique, Hospices Civils de Lyon

M Hermier, J-C Froment, Service de Neuroradiologie, Hôpital Neurologique, Lyon

J-B Pialat, M Wiart, Y Berthezène, CREATIS UMR CNRS 5515, Lyon

Competing interests: none declared

\section{REFERENCES}

1 Hornig CR, Dorndorf W, Agnoli AL. Hemorrhagic cerebral infarction: a prospective study. Stroke 1986;17:179-85.

2 Bozzao L, Angeloni U, Bastianello S, et al. Early angiographic and CT findings in patients with hemorrhagic infarction in the distribution of the middle cerebral artery. Am J Neuroradiol 1991;12:1115-21.

3 Moulin T, Crepin-Leblond T, Chopard JL, et al. Hemorrhagic infarcts. Eur Neurol 1994:34:64-77.

4 Toni D, Fiorelli M, Bastianello S, et al. Hemorrhagic transformation of brain infarct: predictability in the first 5 hours from stroke onset and influence on clinical outcome. Neurology 1996;46:341-5.

5 The NINDS t-PA Stroke Study Group. Intracerebral hemorrhage after intravenous t-PA therapy for ischemic stroke. Stroke 1997;28:2109-18.

6 Larrue V, von Kummer R, del Zoppo G, et al. Hemorrhagic transformation in acute ischemic stroke. Potential contributing factors in the European Cooperative Acute Stroke Study. Stroke 1997;28:957-60.

7 Larrue V, von Kummer R, Müller A, et al. Risk factors for severe hemorrhagic transformation in ischemic stroke patients treated with recombinant tissue plasminogen activator. A secondary analysis of the European-Australasian Acute Stroke Study (ECASS II). Stroke 2001;32:438-41

8 Hacke W, Kaste $M$, Fieschi $C$, et al. Intravenous thrombolysis with recombinant tissue plasminogen activator for acute hemispheric stroke. JAMA 1995;274:1017-26

9 Berger C, Fiorelli M, Steiner T, et al. Hemorrhagic transformation of ischemic brain tissue. Asymptomatic or symptomatic? Stroke 2001;32:1330-35.

10 Tong DC, Adami A, Moseley ME, et al. Relationship between apparent diffusion coefficient and subsequent hemorrhagic transformation following acute ischemic stroke. Stroke 2000;31:2378-84.

11 Tong DC, Adami A, Moseley ME, et al. Prediction of hemorrhagic transformation following acute stroke: role of diffusion- and perfusionweighted magnetic resonance imaging. Arch Neurol 2001;58:587-93.

12 Selim M, Fink JN, Kumar S, et al. Predictors of hemorrhagic transformation after intravenous recombinant tissue plasminogen activator: prognostic value of the initial apparent diffusion coefficient and diffusion-weighted lesion volume. Stroke 2002;33:2047-52.

13 Kidwell CS, Saver JL, Villablanca JP, et al. Magnetic resonance imaging detection of microbleeds before thrombolysis. An emerging application. Stroke 2002;33:95-8.

14 Brott TG, Adams HP, Olinger CP, et al. Measurements of acute cerebral infarction: a clinical examination scale. Stroke 1989:20:864-70.

15 Van Swieten JC, Koudstaal PJ, Visser MC, et al. Interobserver agreement for the assessment of handicap in stroke patients. Stroke 1988;19:604-7.

16 Trouillas $P$, Nighoghossian N, Derex L, et al. Thrombolysis with intravenous rtPA in a series of 100 cases of acute carotid territory stroke. Determination of etiological, topographic and radiological outcomes factors. Stroke 1998;29:2529-40

17 Fazekas F, Kleinert R, Roob G, et al. Histopathologic analysis of foci of signal loss in gradient-echo T2*-weighted MR images in patients with spontaneous intracerebral hemorrhage: evidence of microangiopathy-related microbleeds. Am J Neuroradiol 1999;20:637-42.
18 Longstreth WT, Bernick C, Manolio TA, et al. Lacunar infarcts defined by magnetic resonance imaging of 3660 elderly people: the Cardiovascular Health Study. Arch Neurol 1998;55:1217-25.

19 Fazekas F, Chawluk JB, Alavi A, et al. MR signal abnormalities at 1.5T in Alzheimer's dementia and normal aging. AJNR Am J Neuroradiol 1987:8:421-6

20 Adams HP, Bendixen BH, Kappelle $\sqcup$, et al. Classification of subtype of acute ischemic stroke. Definitions for use in a multicenter clinical trial. TOAST. Trial of Org 10172 in Acute Stroke Treatment. Stroke 1993;24:35-41.

21 Furlan A, Higashida R, Wechsler L, et al. Intra-arterial prourokinase for acute ischemic stroke. The PROACT II Study: a randomized controlled trial. JAMA 1999;282:2003-11

22 Hermier M, Nighoghossian N, Adeleine $P$, et al. Early magnetic resonance imaging prediction of arterial recanalization and late infarct volume in acute carotid artery stroke. J Cereb Blood Flow Metab 2003;23:240-8.

23 Rohl R, Ostergaard L, Simonsen CZ, et al. Viability thresholds of ischemic penumbra of hyperacute stroke defined by perfusion-weighted MRI and apparent diffusion coefficient. Stroke 2001;32:1140-6.

24 Schellinger PD, Fiebach JB, Hacke W. Imaging-based decision making in thrombolytic therapy for ischemic stroke: present status. Stroke 2003;34:575-83

25 Wardlaw JM, del Zoppo G, Yamaguchi T. Thrombolysis for acute ischaemic stroke (Cochrane review). In: The Cochrane Library. Oxford, UK: Update Software, 2002

26 Dijkhuizen RM, Asahi M, Wu O, et al. Rapid breakdown of microvascular barriers and subsequent hemorrhagic transformation after delayed recombinant tissue plasminogen activator treatment in a rat embolic stroke model. Stroke 2002;33:2100-4.

27 Ueda T, Hatakeyama T, Kumon Y, et al. Evaluation of risk of hemorrhagic transformation in local intra-arterial thrombolysis in acute ischemic stroke by initial SPECT. Stroke 1994;25:298-303.

28 Von Kummer R, Meyding-Lamade U, Forsting M, et al. Sensitivity and prognostic value of early $\mathrm{CT}$ in occlusion of the middle cerebral artery trunk. Am J Neuroradiol 1994;15:9-15

29 Tomsick T, Brott T, Barsan W, et al. Prognostic value of the hyperdense middle cerebral artery sign and stroke scale score before ultraearly thrombolytic therapy. Am J Neuroradiol 1996;17:79-85.

30 Petitti N. The hyperdense middle cerebral artery sign. Radiology 1998;208:687-8.

31 Del Zoppo GJ, von Kummer R, Hamann GF. Ischaemic damage of brain microvessels: inherent risks for thrombolytic treatment in stroke. J Neurol Neurosurg Psychiatry 1998;65:1-9.

32 Tanne D, Kasner SE, Demchuk AM, et al. Markers of increased risk of intracerebral hemorrhage after intravenous recombinant tissue plasminogen activator therapy for acute ischemic stroke in clinical practice: the Multicenter rt-PA Stroke Survey. Circulation 2002;105:1679-85.

33 The Stroke Prevention in Reversible Ischemia Trial (SPIRIT) Study Group. A randomized trial of anticoagulants versus aspirin after cerebral ischemia of presumed arterial origin. Ann Neurol 1997;42:857-65.

34 Nighoghossian $N$, Hermier $M$, Adeleine $P$, et al. Old microbleeds are a potential risk factor for cerebral bleeding after ischemic stroke: a gradientecho T2*-weighted brain MRI study. Stroke 2002;33:735-42.

35 Demchuk AM, Morgenstern LB, Krieger DW, et al. Serum glucose level and diabetes predict tissue plasminogen activator-related intracerebral hemorrhage in acute ischemic stroke. Stroke 1999;30:34-9.

36 Lindsberg PJ, Soinne L, Roine RO, et al. Community-based thrombolytic therapy of acute ischemic stroke in Helsinki. Stroke 2003;34:1443-9.

37 Gisselsson L, Smith ML, Siesjo BK. Hyperglycemia and focal brain ischemia. J Cereb Blood Flow Metab 1999;19:288-97.

38 Hamann GF, del Zoppo GJ, von Kummer R. Hemorrhagic transformation of cerebral infarction: possible mechanisms. Thromb Haemost 1999:82(suppl 1):92-4.

39 Hart RG, Easton JD. Hemorrhagic infarcts. Stroke 1986;17:586-9. 